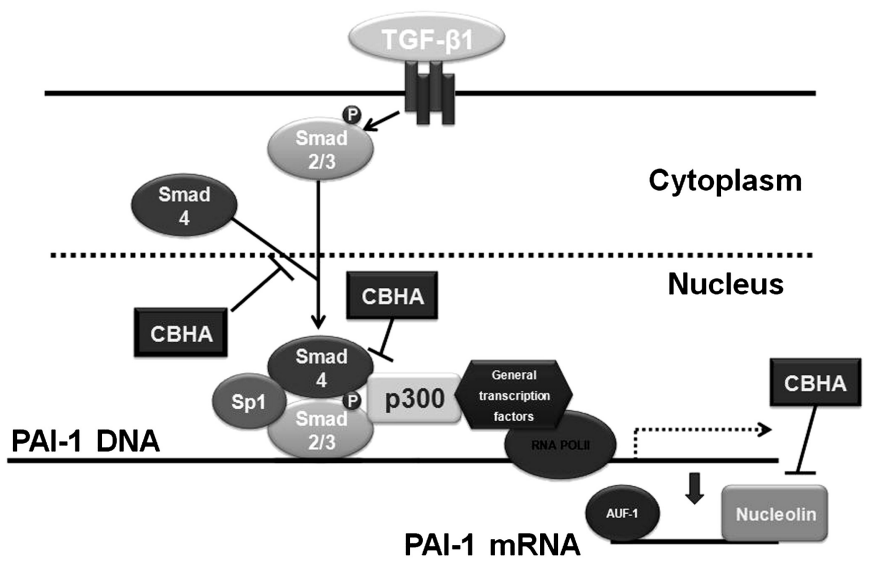

Abstract P66 Figure 1

Conclusion Our data suggests that inhibition of HDAC activity by CBHA may attenuate TGF- $\beta 1$-induced PAI- 1 expression in human pleural mesothelial cells through modulation of cellular signaling at multiple levels. HDAC inhibitors may be employed as potential therapeutic agents for pleural fibrosis.

\section{P67 OUTCOMES FROM SURGICAL MANAGEMENT OF PLEURAL INFECTION: 12-YEAR EXPERIENCE FROM A TERTIARY CARDIOTHORACIC CENTRE}

doi:10.1136/thx.2010.150979.18

${ }^{1} \mathrm{D} J \mathrm{~B}$ Marks, ${ }^{2} \mathrm{M}$ Fisk, ${ }^{1} \mathrm{C}$ Y Koo, ${ }^{2} \mathrm{~S} F$ Lee, ${ }^{2} \mathrm{D}$ Lawrence, ${ }^{1} \mathrm{R} \mathrm{F}$ Miller, ${ }^{1} \mathrm{~A}$ Zumla. ${ }^{1}$ University College London, London, UK; ${ }^{2}$ Heart Hospital, London, UK

Introduction and Objectives Thoracic empyema affects $>65000$ patients/year in the US and UK. Up to $50 \%$ require surgical drainage, and $15 \%$ die. We describe clinical features, microbiology, risk factors and surgical outcomes from referrals to a regional specialist cardiothoracic centre over 12 year.

Methods Patients were identified by searching the hospital Clinical Data Repository for a diagnosis of 'pyothorax' from 1999 to 2010. A retrospective observational study was conducted using case note and database review.

Results 406 distinct empyemas were identified. Patients were predominantly male ( $\mathrm{n}=301,74.1 \%$ ), with median age of 53 years $(\mathrm{IOR}=37-69)$. Pyothorax predominantly developed secondary to community-acquired pneumonia, although 51 (12.6\%) were hospitalacquired; $70(17.2 \%)$ were receiving steroids or immunosuppressants, $35(8.6 \%)$ had concurrent malignancy, 33 (8.1\%) were diabetic and 14 (3.4\%) patients were HIV-infected. Empyemas were right-sided in 233 $(57.3 \%, p=0.03)$; four were bilateral. A causative organism was identified in $229(56.4 \%)$ patients. Positive cultures were obtained from sputum in $59(14.5 \%)$, pleural fluid in $174(42.9 \%)$ and blood in 61 $(15.0 \%)$ patients. Organisms identified included Streptococcus milleri $(\mathrm{n}=18,4.4 \%)$, Streptococcus pneumoniae $(\mathrm{n}=39,9.6 \%)$, other streptococci $(n=10,2.5 \%)$, Enterobacteraciae $(n=12,3.0 \%)$, anaerobes $(n=32$, 7.9\%), methicillin-sensitive Staphylococcus aureus ( $\mathrm{n}=36,8.9 \%$ ), methicillin-resistant Staphylococcus aureus $(\mathrm{n}=25,6.2 \%)$, Enterococcus $(\mathrm{n}=14,3.4 \%)$, Mycobacterium tuberculosis ( $\mathrm{n}=36,8.9 \%$ ), non-tuberculous mycobacteria $(n=1,0.2 \%)$, other bacteria $(n=25,6.2 \%)$, and fungi ( $n=9,2.2 \%) ; 34(8.4 \%)$ cultures were polymicrobial. $277(68.2 \%)$ patients were managed by open thoracotomy. 116 (28.6\%) underwent video-assisted thoracoscopic surgery (VATS), of whom 17 (14.7\%) were converted to open. Three $(0.7 \%)$ required a Clagett window; 10 $(2.5 \%)$ were managed with tube thoracostomy alone. A significant trend towards increased use of VATS over time was noted $(\mathrm{p}=0.0002)$. All-cause complication rate was $20.7 \%$, and 28 -day mortality was $5.7 \%$. Low preoperative haemoglobin was predictive of mortality $(p=0.04)$, but admission and peak C-reactive protein were not.
Conclusions In this large series of empyemas from a tertiary referral centre, microbiological diagnosis was achieved in only half the patients. The identification of tuberculosis in 36 cases means routine screening for mycobacteria is necessary. Use of VATS has increased significantly, and mortality was lower than previously reported and associated with preoperative anaemia.

Contributors DJBM and MF contributed equally to this work.

\section{P68 SURGERY FOR MESOTHELIOMA: THE CASE FOR MACROSCOPIC COMPLETE RESECTION}

doi:10.1136/thx.2010.150979.19

K K W Lau, A Nakas, D A Waller. Glenfield Hospital, Leicester, UK

Objective The role for radical surgery for Malignant Pleural Mesothelioma (MPM) remains controversial. There are advocates of less radical 'debulking surgery' who argue against the need for removal of diaphragm and pericardium because of increased morbidity. We test the hypothesis that survival is increased by the more radical operations intended to remove all visible tumour and achieve macroscopic complete resection (MCR).

Methods Over a 13-year period, 362 patients underwent therapeutic surgery for MPM: in-group MCR: 232 (64\%) patients underwent either extra-pleural pneumonectomy (135 patients $(37 \%)$ ) or radical pleurectomy-decortication (97 patients $(27 \%)$ ) with resection of diaphragm and pericardium. Whilst in group D: 130 (36\%) underwent debulking surgery leaving macroscopic tumour in situ (55 patients $(15 \%)$ by thoracotomy and 75 patients $(21 \%)$ by VATS). The patients in the MCR group were younger (mean age 57.9 vs $66.1, \mathrm{p}<0.0001)$ and more had epithelioid subtype (167 (72\%) vs 86 $(66.2 \%), p<0.0001)$. We compared the hospital outcomes and overall survival between these two groups.

Results The mean length of stay was longer in group MCR than in group D (16.0 days vs 9.6 days, $\mathrm{p}<0.0001)$ but 30 -day mortality was similar $(12(5.2 \%)$ vs $8(6.2 \%), p=0.811)$. In univariate analysis, overall survival was significantly longer in the MCR group (mean survival 22 months vs 13 months, $\mathrm{p}<0.0001$ ). A similar benefit was observed for the epithelioid pathological subgroup (mean survival 25.8 months vs 16.6 months, $p<0.0001)$. Cox regression showed that surgery intended to achieve MCR was associated with a significantly reduced hazard of death after adjusting for age and pathological subtype (HR $0.72,95 \%$ CI 0.55 to $0.93, p=0.014$ ).

Conclusions Surgery to increase survival in mesothelioma should be based on an intent to achieve macroscopic complete resection. This strategy should form the basis of future trials to evaluate the role of surgery in this disease.

\section{P69 MESOTHELIOMA IN THE 21ST CENTURY. A 1-YEAR SURVEY OF MALIGNANT MESOTHELIOMA IN THE SOUTH WEST OF THE UK}

doi:10.1136/thx.2010.150979.20

R Bhatnagar, S Earl, K Lansdell, T J Howell. Derriford Hospital, Plymouth, UK

Introduction Malignant mesothelioma $(\mathrm{MM})$ is a cancer that most commonly arises within the pleura, and carries a poor prognosis. There is a strong association with past asbestos exposure so incidence varies across the UK. In the South West of the UK there is a high incidence with many cases being due to asbestos exposure in the ship-building industry.

Aims To describe the demographics, treatment strategies and survival of patients presenting with MM across the Peninsula cancer network, a population of around 1.7 million people, over a 1-year period.

Method Cases of MM diagnosed in 2007 were identified retrospectively and reviewed following interrogation of cancer, MDT, 